\title{
Dopamine Transporter (SLC6A3) Genotype Impacts Neurophysiological Correlates of Cognitive Response Control in an Adult Sample of Patients with ADHD
}

\author{
Thomas Dresler ${ }^{1,5, *}$, Ann-Christine Ehlis ${ }^{1,4,5}$, Sebastian Heinzel',2, Tobias J Renner ${ }^{3}$, Andreas Reif', \\ Christina G Baehne', Monika Heine', Andrea Boreatti-Hümmer', Christian P Jacob', Klaus-Peter Lesch', \\ Andreas J Fallgatter ${ }^{4}$ \\ 'Department of Psychiatry, Psychosomatics and Psychotherapy, University Hospital Wuerzburg, Wuerzburg, Germany; ${ }^{2}$ Department of Genetics \\ and Neurobiology, Theodor-Boveri-Institute, University of Wuerzburg, Wuerzburg, Germany; ${ }^{3}$ Department of Child and Adolescent Psychiatry, \\ Psychosomatics and Psychotherapy, University Hospital Wuerzburg, Wuerzburg, Germany; ${ }^{4}$ Department of Psychiatry and Psychotherapy, \\ University of Tuebingen, Tuebingen, Germany
}

\begin{abstract}
Studies provide ample evidence for a dysfunction in dopaminergic neurotransmission in Attention-Deficit/Hyperactivity Disorder (ADHD). In that respect, a common variable number of tandem repeats (VNTR) polymorphism in the $3^{\prime}$ untranslated region (UTR) of the dopamine transporter gene (SLC6A3) has been repeatedly associated with the disorder. Here, we examined the influence of the common 9- and 10-repeat alleles of SLC6A3 on prefrontal brain functioning and cognitive response control in a large sample of adult ADHD patients $(n=|6|)$ and healthy controls $(n=109)$. To this end, we inspected a neurophysiological marker of cognitive response control (NoGo anteriorization, NGA) elicited by means of a Go-NoGo task (continuous performance test, CPT). Within the group of ADHD patients, nine-repeat allele carriers showed significantly reduced NGA, whereas no influence of SLC6A3 genotype was observed in the control group. In contrast to previous association studies of children, the nine-repeat — not the 10-repeat_-allele was associated with functional impairments in our sample of adult ADHD patients. Our findings confirm a significant effect of the SLC6A3 genotype on the neurophysiological correlates of cognitive response control in ADHD, and indicate that still to-be-identified age-related factors are important variables modulating the effect of genetic factors on endophenotypes. Neuropsychophamacology (2010) 35, 2193-2202; doi:I0.1038/npp.2010.91; published online 14 July 2010
\end{abstract}

Keywords: ADHD; dopamine; SLC6A3; continuous performance test; NoGo anteriorization; imaging genetics

\section{INTRODUCTION}

Attention-Deficit/Hyperactivity Disorder (ADHD) is one of the most prevalent psychiatric disorders in childhood (Biederman and Faraone, 2005), which - in contrast to previous assumptions - often persists into adolescence and adulthood (Barkley, 1998; Simon et al, 2009). A plethora of family, adoption, and twin studies show that heritability has a major role (see references Biederman, 2005; Faraone et al, 2005; Faraone and Doyle, 2000; Martin et al, 2002) as up to $80 \%$ of the phenotypic variability can be explained by genetic influence (eg, Faraone and Doyle, 2000; Levy et al, 1997). Results from molecular genetic studies support a complex genetic architecture of $\mathrm{ADHD}$, that is, genetic

\footnotetext{
*Correspondence: T Dresler, University Hospital Wuerzburg, Psychophysiology and Functional Imaging, Fuechsleinstrasse 15, Wuerzburg 97080, Germany, Tel: + 49 931 201 77410, Fax: + 49 931 20177550, E-mail: Dresler_T@klinik.uni-wuerzburg.de

${ }^{5}$ These authors contributed equally to this work.

Received 16 February 2010; revised 26 May 2010; accepted 4 June 2010
}

vulnerability is mediated by a multitude of risk genes with small individual effects (Faraone et al, 2005). One of the most extensively studied genes in (childhood) ADHD is SLC6A3, the gene encoding the dopamine transporter (DAT). DAT regulates the reuptake of dopamine from the extracellular space, thereby terminating its synaptic action. A genetic polymorphism identified as a potential risk factor for $\mathrm{ADHD}$ is a $40-\mathrm{bp}$ variable number of tandem repeats (VNTR) polymorphism within the $3^{\prime}$ untranslated region (UTR) of SLC6A3 (Vandenbergh et al, 1992). It has two common alleles designated as nine-repeat and 10-repeat, which have been suggested to influence SLC6A3 expression and, thereby, dopamine regulation (Mill et al, 2002; vanNess et al, 2005; Fuke et al, 2001; Miller and Madras, 2002; Greenwood and Kelsoe, 2003 Michelhaugh et al, 2001). However, these in vitro findings concerning the effect of the two allelic variants on SLC6A3 expression remain inconsistent with partly contradicting results.

Meta-analyses of genetic association studies indicate an overrepresentation of the 10-repeat allele in children with ADHD (Faraone et al, 2005). However, findings are 
heterogeneous and may depend on the specific statistical analysis method (Todd et al, 2005; Li et al, 2006; Yang et al, 2007; Langley et al, 2005; Cheuk et al, 2006). Furthermore, in adult samples, the nine-repeat allele has been shown to be associated with the disease, suggesting a differential association depending on age, and a role of SLC6A3 in modulating the ADHD phenotype over life time (Franke et al, 2008, 2010).

These inconsistent findings emphasize the necessity to not only identify potential risk genes for ADHD, but also specific mechanisms by which genetic variants affect the risk for disease. A promising approach might therefore be to relate candidate polymorphisms to specific behavioral, cognitive, or neurophysiological phenotypes. These socalled 'endophenotypes' are considered to be more directly connected to the relevant underlying psycho- or neuropathology - and thereby etiological factors - than categorical clinical diagnoses. Response inhibition, which has a key role in many of the neuropsychological models (see Barkley, 1997, 1998), has been suggested as a possible endophenotype for ADHD (Sonuga-Barke, 2002; see also reference Crosbie et al, 2008). Whereas brain-imaging studies in ADHD confirm functional deficits in areas underlying inhibitory control (eg, Rubia et al, 1999; Smith et al, 2006), current concepts additionally consider other aspects of prefrontal response control, for example, higherorder controlled motor function, attentional control, and mental flexibility (Slaats-Willemse, 2003). Paradigms involving one or more of these processes therefore seem to be particularly suited to study altered cognitive functioning in $\mathrm{ADHD}$ and the effect of genetic variants on neural processing.

Behavioral studies in children report a detrimental effect of the 10-repeat allele on neurocognitive performance in vigilance and attention tasks (Loo et al, 2003; Bellgrove et al, 2005). However, Barkley et al (2006) conducted a longitudinal study on children, adolescents, and young adults with $\mathrm{ADHD}$, and report greater $\mathrm{ADHD}$ and externalizing symptoms, as well as family, educational, and occupational impairments at all three follow-up examinations in 9/10- as compared with that in 10/10repeat allele carriers. Also, 9/10-repeat carriers in the control group were prone to higher impairment on educational, occupational, and neurocognitive measures.

Neurophysiological measures might have the advantage of being even more closely linked to neurobiological mechanisms than behavioral measures alone ('Imaging Genetics' approach; Fallgatter et al, 1999a; Bookheimer et al, 2000; Ehlis et al, 2007; Egan et al, 2001). In the present investigation, we therefore used a topographical ERP parameter (NoGo anteriorization/NGA), which has been proposed to reflect the mechanisms of prefrontal response control, to examine a group of adult ADHD patients and age-matched healthy controls stratified for the SLC6A3 genotype (40-bp VNTR within the $3^{\prime}$ UTR). Subjects performed a Go-NoGo task (Continuous Performance Test/CPT; Rosvold et al, 1956), which involves processes of response inhibition and response execution (higherorder motor and attention control). For this paradigm the NGA has been established as a reliable marker reflecting medial prefrontal functioning (Fallgatter et al, 1997; Fallgatter and Strik, 1999). In schizophrenic patients the
NGA was found to be related to variants of the catechol$O$-methyltransferase (COMT) gene (Ehlis et al, 2007). Furthermore, in one of our previous publications, the tryptophan hydroxylase (TPH2) gene was found to have a significant effect on the NGA in adult ADHD patients (Baehne et al, 2009). In this study, we hypothesized that the SLC6A3 genotype would significantly affect the NGA, particularly in ADHD patients. Because of recent results suggesting a specific role of the SLC6A3 nine-repeat allele in adult patients with ADHD (Franke et al, 2010), we furthermore assumed that the NGA would be particularly altered in adult ADHD patients carrying at least one ninerepeat allele of the VNTR.

\section{MATERIALS AND METHODS}

\section{Participants}

A total of 161 adult patients, diagnosed with ADHD (DSM-IV criteria), participated in the study. They were recruited through the in- and outpatient facilities of the Department of Psychiatry, Psychosomatics and Psychotherapy of the University of Wuerzburg. One hundred and nine healthy subjects without history of psychiatric and neurological disorders were recruited from the local region. All participants gave written informed consent after complete description of the study. Exclusion criteria were age below 18 and above 60 years, IQ below 90, current psychotropic medication, as well as serious somatic or neurological disorders. A total of 171 participants (94 ADHD patients, 77 controls) were also included in one of our previous publications (Baehne et al, 2009). All subjects were of Caucasian origin.

Participants were stratified according to their SLC6A3 $3^{\prime}$ UTR VNTR genotype into a homozygous 10-repeat allele group (10/10) and a group carrying at least one nine-repeat allele $(9 / 9$ or $9 / 10)$. The $10 / 10$ group comprised 84 patients and 57 controls, the other genotype group 77 patients (9/9: $n=9 ; 9 / 10: n=68)$ and 52 controls (9/9: $n=8$; 9/10: $n=44)$. Descriptive statistics are displayed in Table 1. Genotype frequencies (9/9 \& 9/10 vs 10/10) did not differ between diagnostic groups $\left(\chi_{1}^{2}<0.01, p=0.99\right)$. Ten-repeat allele frequencies were in Hardy-Weinberg equilibrium for both patients $\left(\chi_{1}^{2}=1.00, p=0.32\right)$ and controls $\left(\chi_{1}^{2}=0.02, p=0.90\right)$. Gender ratios did not differ between genotypes (ADHD: $\chi_{1}^{2}=0.05, p=0.82$; control group: $\left.\chi_{1}^{2}=2.59, p=0.11\right)$. Lefthandedness was overrepresented in $9 / 9$ and $9 / 10$ carriers with $\operatorname{ADHD}\left(\chi_{1}^{2}=6.82, p=0.01\right)$, whereas no differences were found in the control group $\left(\chi_{1}^{2}=0.26, p=0.61\right.$; see Table 1$)$. For IQ scores (for 10 subjects (4 patients/6 controls) no IQ data were available), as assessed with the MWT-B (Lehrl, 2005), a measure of crystallized verbal intelligence, a $2 \times 2$ analysis of variance (ANOVA) showed a significant effect for 'diagnosis' $\left(\mathrm{F}_{1,256}=6.10, p=0.01\right)$, with higher scores for the controls but no significant main-effect 'genotype' $\left(\mathrm{F}_{1,256}=0.57, p=0.45\right)$ and no interaction of 'diagnosis $\times$ genotype' $\left(F_{1,256}=0.05, p=0.82\right)$. Neither 'diagnosis' nor 'genotype' had an effect on the distribution of age $\left(\mathrm{F}_{1,266}<1.00, p>0.50\right)$. As expected, 'diagnosis' had a significant effect on the German short-version of the Wender Utah Rating Scale (WURS-k; Retz-Junginger et al, 2002) $\left(\mathrm{F}_{1,260}=218.73, p<0.01\right.$; for a total of six 
Table I Sample Characteristics (Standard Deviation in Parentheses)

\begin{tabular}{|c|c|c|c|c|c|c|}
\hline & \multicolumn{3}{|c|}{ ADHD patients } & \multicolumn{3}{|c|}{ Controls } \\
\hline & $9 / 9 \& 9 / 10(n=77)$ & $10 / 10(n=84)$ & All $(n=161)$ & $9 / 9 \& 9 / 10(n=52)$ & $10 / 10(n=57)$ & All $(n=109)$ \\
\hline IQ & || $3.5 \mid(|| .30)$ & | | 4.37 (13.08) & II $3.96(12.22)$ & $117.10(13.36)$ & I 18.65 (12.67) & $117.93(12.96)$ \\
\hline Handedness (right/left) & $65 / 12$ & $81 / 3$ & $146 / 15$ & $48 / 4$ & $51 / 6$ & $99 / 10$ \\
\hline
\end{tabular}

patients, no WURS-k score was available due to single items missing), with ADHD patients scoring higher than control participants, whereas 'genotype' and interaction of 'diagnosis $\times$ genotype' had no significant influence $\left(\mathrm{F}_{1,260}<0.25, p>0.6\right)$.

According to DSM-IV, 99 patients were classified as the combined subtype, 43 as the inattentive subtype, and 10 as the hyperactive/impulsive subtype of ADHD. Subtype composition (for nine patients subtype diagnosis was missing) did not differ significantly between the two genotype groups $\left(\chi_{2}^{2}=0.84, p=0.66\right)$. Regarding current psychiatric comorbidities, as assessed with the Structured Clinical Interview for DSM-IV (SCID-I, Wittchen et al, 1997), $47 \%$ of the patients showed a comorbid axis-I disorder: 19 patients were diagnosed with substance misuse/ dependency ( 8 for the 9/9 \& 9/10 genotype $v s 11$ for the 10/10 genotype; specific diagnoses: alcohol abuse (F10.1; $n=2)$ or dependency (F10.2; $n=2)$; cannabinoid abuse $(\mathrm{F} 12.1 ; n=3)$ or dependency $(\mathrm{F} 12.2 ; n=12)), 32$ with mood disorders (18 vs 14; specific diagnoses: bipolar affective disorders (F31.0, F31.8; $n=5)$, depressive episodes (F32.1, F32.8; $n=3$ ), recurrent depressive episodes (F33.0, F33.1; $n=8)$, cyclothymia (F34.0; $n=2)$, dysthymia (F34.1; $n=7)$, and other recurrent mood disorders (F38.1; $n=7)$ ) and 25 with neurotic, stress-related, and somatoform disorders (14 vs 11; specific diagnoses: agoraphobia (F40.0; $n=1$ ), social phobias (F40.1; $n=10)$, specific phobias (F40.2; $n=6)$; panic disorder (F41.0; $n=1)$; generalized anxiety disorder (F41.1; $n=2)$, obsessive-compulsive disorder (F42.0; $n=1)$, post-traumatic stress disorder (F43.1; $n=2)$, bulimia nervosa (F50.2; $n=1)$, and unspecified eating disorders (F50.9; $n=1)$ ). Regarding smoking status, 62 ADHD patients (38.5\%) and 17 healthy controls (15.6\%) were daily tobacco smokers $\left(\chi_{1}^{2}=16.49, p<0.001\right)$. The study was reviewed and approved by the Ethics Committee of the University of Wuerzburg, and the procedures involved were in accordance with the Declaration of Helsinki.

\section{SLC6A3 Genotyping}

Genomic DNA was extracted from whole-blood samples by salt precipitation according to standard protocols. The SLC6A3 3' UTR VNTR was genotyped by PCR and subsequent gel electrophoresis as published previously (Hünnerkopf et al, 2007). Further details on protocols are available upon request.

\section{Electrophysiological Investigation}

The participants performed an OX-version of the Continuous Performance Test (Rosvold et al, 1956). The measurements took place in a dimly lit, quiet room. All the letters were presented sequentially on a computer screen (viewing distance: $80 \mathrm{~cm}$ ) in a pseudo-randomized order. The stimuli were approximately $30 \mathrm{~mm}$ high and $20 \mathrm{~mm}$ wide, resulting in a visual angle of 2.15 degrees vertically and 1.43 degrees horizontally. The patients and controls were instructed to press a response button only when the letter $\mathrm{O}$ was directly followed by the letter X. Speed and accuracy were emphasized equally during the explanation of the test. Before the actual CPT, a short training session was performed. The complete stimulus set consisted of 400 letters (114 letters $\mathrm{O}=$ primer condition, $57 \mathrm{X}$ following an $\mathrm{O}=$ Go condition, 57 other letters following an $\mathrm{O}=$ NoGo condition, and 172 letters not following an $\mathrm{O}=$ distractors) with a stimulus-onset asynchrony (SOA) of $1850 \mathrm{~ms}$ and a stimulus presentation time of $200 \mathrm{~ms}$.

During performance of the task, a continuous EEG was recorded from 21 scalp electrodes placed according to the International 10/20-System (Jasper, 1958). Three additional electrodes were attached at the outer canthi of both eyes and below the right eye for registration of eye movements. The technical equipment consisted of a 32-channel DC amplifier and the data acquisition software 'Vision Recorder' (Brain Products, Munich, Germany). The hardware filter was set to a bandpass from 0.1 to $100 \mathrm{~Hz}$ and A/D rate was $1000 \mathrm{~Hz}$. The recording reference was placed between $\mathrm{Fz}$ and $\mathrm{Cz}$ and the ground electrode between $\mathrm{Fpz}$ and Fz. All electrode impedances were kept below $5 \mathrm{k} \Omega$.

\section{Data Analysis}

Electrophysiological data were processed using the 'Vision Analyzer' software (Brain Products, Munich, Germany). In a first step, data were filtered offline with a bandpass from 0.1 to $70 \mathrm{~Hz}$, re-referenced to an average reference, and corrected for ocular artifacts using the standard algorithm implemented in the software (Gratton and Coles, 1989). After a computerized artifact rejection (only amplitudes $<70 \mu \mathrm{V}$ in all EEG channels within $100 \mathrm{~ms}$ before and $700 \mathrm{~ms}$ after stimulus presentation were allowed) artifactfree epochs after correct responses were segmented and individually averaged to Go and NoGo event-related potentials (ERPs). On the basis of the centroid method 


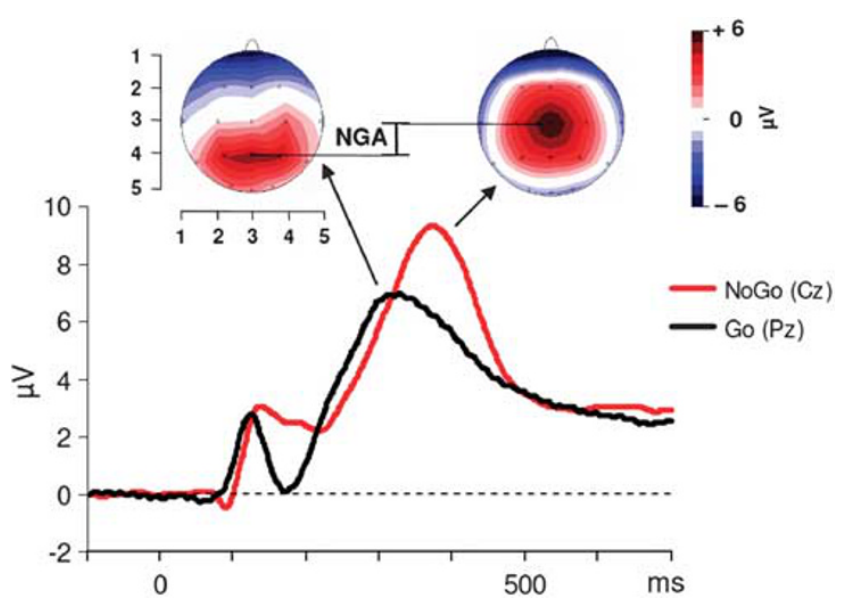

Figure I A schematic illustration of the quantification of the NGA as the geometrical distance between Go and Nogo centroid in the anteriorposterior direction. Grand average curves of Go (Pz; black line) and Nogo ( $\mathrm{Cz}$; red line) trials for the group of healthy controls homozygous for the I0-repeat allele. The heads illustrate the distribution of the positive brain electrical field at the respective peak of the P300.

proposed by Lehmann (1987), the individual P300 latencies were determined on the basis of the peaks of the global field power (GFP) in a P300 time window of 275-530 ms after stimulus presentation. The GFP is a one-number estimator of the electrical field strength in multi-channel EEG recordings and corresponds to the standard deviation of all measured potential values at a given point of time. At this latency, the two-dimensional topography of both the Go and the NoGo condition was analyzed by means of the area centroids of the P300 field maps. The area centroids are amplitudeweighted locations (centers of gravity) of the positive and the negative areas of the brain electrical field after elimination of the spatial DC offset (average reference). The location of each individual centroid was quantified on an anterior - posterior axis by a coordinate system resulting from the planar projection of the electrode array onto a rectangular grid. Centroids could obtain values between 1 (level of electrode position Fpz) and 5 (level of $\mathrm{Oz}$ ) as illustrated in Figure 1 (locations somewhere in between two electrode positions were expressed by respective decimal numbers). Smaller values of centroid locations indicate a more anterior localization. On the basis of our previous studies (eg, Fallgatter et al, 1997), only the anteriorposterior location of the positive centroid during the Go and NoGo condition was considered in more detail. The individual NGA was calculated as the difference between Go and NoGo centroid on the anterior-posterior axis.

\section{Statistical Analysis}

Statistical analyses were performed with the software SPSS 14.0. For the NGA, a $2 \times 2$ ANOVA was conducted, comprising the between-subject factors 'diagnosis' and 'genotype'. Post-hoc analyses were calculated by means of two-tailed $t$-tests for independent samples for betweengroup comparisons of the NGA as well as Go and NoGo centroids. $t$-tests were used to compare mean Go reaction times between the two genotype groups. Equality of variances was tested by means of Levene's test; corrections for inequality were performed when necessary. As none of the CPT error data were normally distributed according to Kolmogorov-Smirnov's $Z$-statistic ( $p$-values $<0.01$ ), Mann-Whitney $U$-tests were used for between-group comparisons. Except for the Go centroid and the NGA data in the group of ADHD patients with the 9/9 \& 9/10 genotype, all electrophysiological parameters in the four groups were normally distributed. As an ANOVA with a sufficient number of participants per cell is relatively robust against such violations, we decided to apply the $2 \times 2$ ANOVA approach to our data. The significance level was set to $p<0.05$.

To conduct exploratory analyses on any allele-dose effects, the group comprising the $9 / 9$ and $9 / 10$ genotype was split into two separate groups, one containing only $9 / 9$ and the other only $9 / 10$ allele carriers. Despite the low number of subjects in the 9/9 group (ADHD: $n=9$, controls: $n=8$ ), we opted for an-at least descriptive-analysis of these subgroups. To this end, we applied a trend test, assuming a linear relationship of the three genotypes $(9 / 9$, $9 / 10,10 / 10)$ regarding their effect on the electrophysiological data.

Furthermore, to conduct exploratory analyses we applied source localization with the sLoreta software (PascalMarqui et al, 1994; Pascual-Marqui, 2002; Pascual-Marqui et $a l, 2002)$ to examine the source of the topographical EEG markers.

\section{RESULTS}

\section{Behavioral Data}

ADHD patients made more commission errors after primers and distractors (Mann-Whitney $U=6565.0, Z=-3.90$, $p<0.01)$, more omission errors $(U=6610.5, Z=-3.72$, $p<0.01)$, and had elongated reaction times in the Go trials ( $U=6500.0, Z=-3.61, p<0.01)$ as compared with healthy controls. In addition, they showed a greater variability in reaction time than the controls $(U=5980.0, Z=-4.44$, $p<0.01)$. The patients and healthy controls did not differ with respect to the number of commission errors in the NoGo trials $(U=8619.5, Z=-0.46, p=0.65)$. Within the whole sample, no significant behavioral differences were found between the two genetic groups (10/10 vs 9/10 \& 9/9), except for marginally significant differences in the number of commission errors after primers and distractors ( $U=8115.5, Z=-1.70, p=0.09,10 / 10$ group with more errors) and in the number of commission errors in the NoGo trials $(U=8525.0, Z=-1.65, p=0.10,10 / 10$ group with less errors). These trend-like differences could be attributed to corresponding effects in the control group ( $U=1260.0, Z=-1.65, p=0.10$ and $U=1325.5, Z=-1.69$, $p=0.09$, respectively), whereas no genotype differences emerged within the group of ADHD patients $(p>0.20)$. When behavioral data were controlled for the influence of intelligence, which differed between the ADHD patients and the controls (see Materials and Methods), the results remained virtually unchanged. Descriptive statistics of the behavioral data are shown in Table 2. 
Table 2 Descriptive Statistics of Behavioral Data (Standard Deviation in Parentheses)

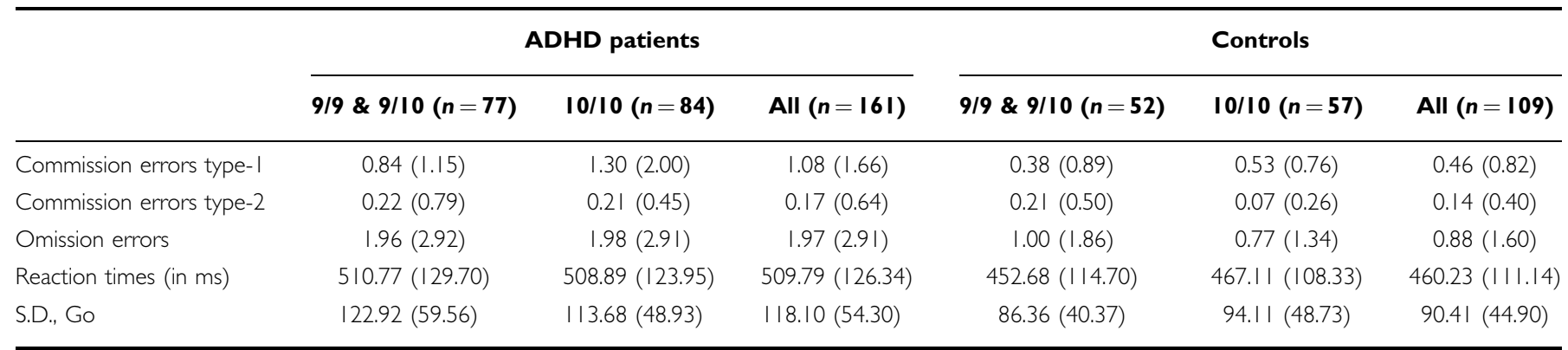

\section{ERP Data}

Concerning the NGA (ie, the difference measure resulting from subtraction of the NoGo from the Go centroid: Centroid $_{\mathrm{Go}}-$ Centroid $_{\mathrm{NoGo}}$ ), the $2 \times 2$ ANOVA conducted to test for effects of the factors 'diagnosis' (ADHD $v s$ control group) and 'genotype' (9/10 \& 9/9 vs 10/10) showed a marginally significant effect for 'diagnosis' $\left(\mathrm{F}_{1,266}=3.53\right.$, $p=0.06)$ and a significant interaction 'diagnosis $\times$ genotype' $\left(\mathrm{F}_{1,266}=6.42, p=0.01\right)$. A tendency of lower NGA values was observed in patients as compared with controls $(0.36 \pm 0.77 v s 0.50 \pm 0.53)$. The interaction effect of 'diagnosis $\times$ genotype' was attributable to the fact that the 3 ' UTR VNTR had a significant effect on the NGA only within the group of adult ADHD patients (see Figure 2): More precisely, patients with the $9 / 9$ or $9 / 10$ genotype showed a significantly diminished NGA as compared with patients carrying the $10 / 10$ genotype $(0.21 \pm 0.78$ vs $\left.0.49 \pm 0.74, t_{159}=2.34, p=0.02\right)$. By contrast, the NGA was not affected by the SLC6A3 genotype in the group of healthy controls $\left(0.58 \pm 0.61\right.$ vs $\left.0.44 \pm 0.43, t_{92}=1.42, p=0.16\right)$. Directly comparing patients and controls for each of the two genotype groups also showed a significant reduction of the NGA in ADHD patients only within the group of 9/9 and $9 / 10$ allele carriers $\left(0.21 \pm 0.78\right.$ vs $0.58 \pm 0.61, t_{127}=2.87$, $p<0.01)$. No significant difference between diagnostic groups was observed in the $10 / 10$ group $(0.49 \pm 0.74 \mathrm{vs}$ $0.44 \pm 0.43, t_{137}=0.56, p=0.58$ ). Subgroup analysis assuming a linear relationship of the three genotypes showed an allele-dose effect of the 10-repeat allele in adult ADHD patients $\left(\mathrm{F}_{1,158}=5.18, p=0.02\right)$, but no significant result for the control group $\left(\mathrm{F}_{1,106}=1.42, p=0.24\right.$; this test examines linear relationships by testing the contrast $\left(\begin{array}{lll}-1 & 0 & +1\end{array}\right)$ weighting individual values according to the number of 10-repeat alleles). Within the group of ADHD patients, we further tested whether a quadratic trend may explain more variance than the linear one (this test examines quadratic linear relationships by testing the contrast $(+1-2+1))$. As this was not the case $\left(\mathrm{F}_{1,158}=0.34\right.$, $p=0.56$ ), a linear model seems to be the best model for ADHD patients (as can be seen in Figure 3). As the diagnostic groups differed in their verbal intelligence scores, we conducted an analysis of covariance (ANCOVA) with the same factors mentioned above, while adding the standardized covariate 'intelligence'. The results remained virtually unchanged, except for the factor diagnosis that now reached statistical significance $\left(\mathrm{F}_{1,273}=4.21, p=0.04\right)$, with smaller values of the NGA in ADHD patients as compared with controls (see Figure 2).

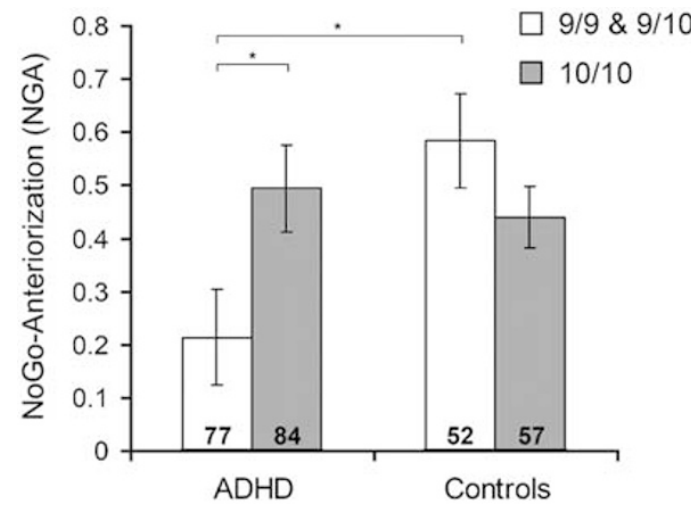

Figure 2 Mean values of the NGA for ADHD patients and healthy controls with either 0 or I 10-repeat allele (white columns) or 2 10-repeat alleles of the DAT gene (gray columns). The error bars represent the standard errors of the mean.

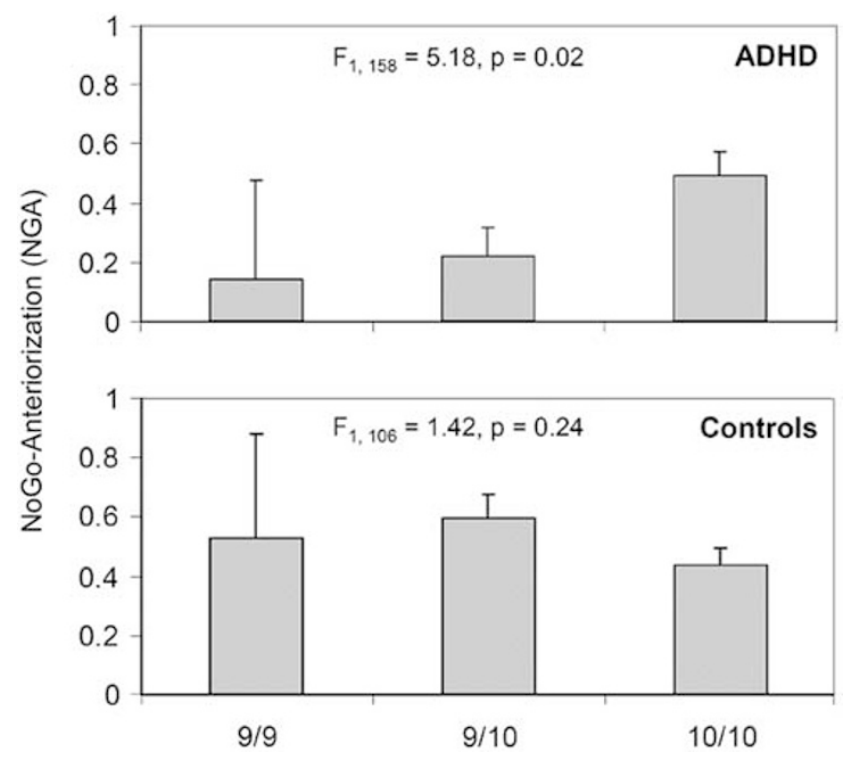

Figure 3 Trend tests to analyze possible allele-dose effects. Mean values of the NGA for ADHD patients (upper panel) and healthy controls (lower panel) stratified according to number of 10 -repeat alleles $(0,1,2)$. The error bars represent the standard errors of the mean.

To further elucidate the origin of the NGA effects in our study, we performed an additional analysis on the positions of the Go and NoGo centroids, both of which contribute to the difference in the measure of the NGA $\left(\mathrm{NGA}=\right.$ Centroid $_{\mathrm{Go}}-$ Centroid $_{\mathrm{NoGo}}$; see above $)$. This analysis 

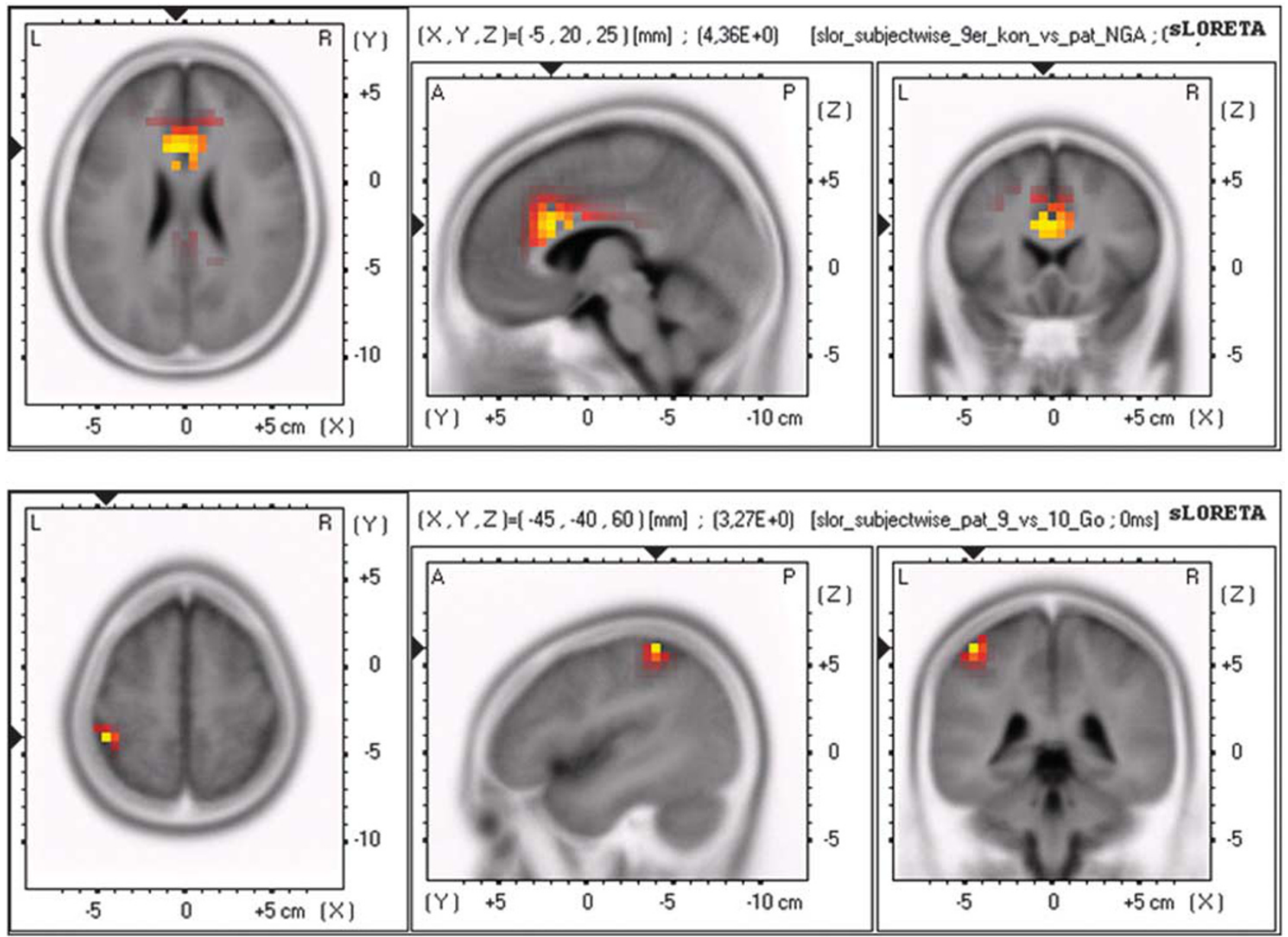

Figure 4 Explorative source localization with sLoreta. The upper panel shows the contrast controls vs ADHD patients for the NGA (NoGo-Go) within the 9/9 \& 9/10 genotype group; the lower panel the contrast 10/10 vs the 9/9 \& 9/10 genotype group for the Go centroid within the patients.

showed that only the Go centroid was significantly affected by the between-subject factors 'diagnosis' and 'genotype'. More precisely, a significant main effect of the factor 'diagnosis' $\left(\mathrm{F}_{1,266}=9.03, p<0.01\right)$ could be explained by a more anterior position of the Go centroid in ADHD patients (mean Go centroid: $3.50 \pm 0.74$ ) as compared with that in healthy controls (mean Go centroid: $3.74 \pm 0.49$ ). Moreover, a significant interaction 'diagnosis $\times$ genotype' occurred $\left(\mathrm{F}_{1,266}=4.25, p<0.05\right)$, indicating that only within the group of ADHD patients carriers of the 9/9 or 9/10 genotype tended to have a more anterior location of the Go centroid (3.40 \pm 0.87$)$ than the carriers of the $10 / 10$ genotype $\left(3.60 \pm 0.57 ; t_{129}=1.76, p<0.1\right)$. In accordance with the results for the NGA reported above, the patients and controls furthermore differed only within the group of the $9 / 9$ and $9 / 10$ allele carriers, with a more anterior location of the Go centroid in ADHD patients ( $3.40 \pm 0.87)$ as compared with that in healthy controls $\left(3.80 \pm 0.50 ; t_{124}=3.35\right.$, $p<0.01$ ). For $10 / 10$ allele carriers, no significant effect of the factor 'diagnosis' was observed $\left(t_{139}=0.82, p=0.41\right)$.

Explorative analyses with sLoreta software were applied to examine the sources of the topographical ERP findings. The source localization indicated that in all genetic subgroups the NGA could be localized to the anterior cingulate cortex. Furthermore, for this contrast (NoGo-Go), healthy subjects with the $9 / 9$ or $9 / 10$ genotype showed a significantly higher recruitment of cingulate cortices as compared with ADHD patients of the same genotype (Figure 4, upper panel). However, for the contrast of the two genotypes within the group of ADHD patients we did not find significant source differences, despite the NGA findings reported above. As our topographic results were mainly driven by the Go centroid, an additional sLoreta analysis was conducted to compare the sources for the Go condition within our group of ADHD patients: Here, a marginally significant difference between genetic groups was found that located a difference source in the left inferior parietal cortex, with higher activation in the $10 / 10$ group (Figure 4, lower panel).

\section{DISCUSSION}

The present EEG study was conducted to further elucidate the influence of the common and functionally relevant 40-bp $3^{\prime}$ UTR VNTR in the dopamine transporter gene SLC6A3 on neurophysiological functioning in adult patients with ADHD. To this end, a large sample of ADHD patients was compared with a healthy control sample regarding their neural responses to a common behavioral inhibition task 
(Continuous Performance Test/CPT). The 'endophenotype approach' in psychiatry (Almasy and Blangero, 2001) assumes that neuropsychological and other functional concepts (eg, behavioral inhibition, attentional control, mental flexibility; see reference Slaats-Willemse, 2003) are more closely related to etiological factors (eg, genes) than categorical clinical diagnoses. Research on functional endophenotypes might therefore aid a more thorough understanding of a disorder, thereby improving current therapeutic options.

Behavioral data showed that ADHD patients performed worse than healthy controls as indicated by the number of commission errors after primers and distractors, number of omission errors, reaction time in Go-trials, and the variability of the reaction time, which is in line with previous findings (eg, Epstein et al, 1998; Leth-Steensen et al, 2000). These findings imply that ADHD patients are less sensitive to stimulus features, that is, to the difference between targets and non-targets, than their normal counterparts (for a meta-analysis see reference Losier et al, 1996). From this point our behavioral data are well in line with the existing literature on CPT performance in $\mathrm{ADHD}$ patients. Regarding the genetic analyses, no significant association of SLC6A3 genotype with behavioral data was found. This is in line with the results obtained by Barkley et al (2006) who implemented a similar task, but does not replicate findings by Bellgrove et al (2005) and Loo et al (2003) who report an influence of the $3^{\prime}$ UTR VNTR on behavioral measures in a Go-NoGo task, especially on response variability. In these previous studies, homozygous 10-repeat allele carriers showed poorer performance and showed greater response time variability than children with at least one nine-repeat allele. Some methodological differences between studies might account for these diverging findings (eg, differences in mean age (children $v s$ adults) and sample size, as well as task specifications).

In the ERP data we found that ADHD patients showed a tendency toward smaller values of the NGA (difference Gominus NoGo centroid) than healthy control subjects $(p=0.06$, in the ANCOVA $p=0.04$; see Figure 2$)$. This finding is in line with the known deficits of inhibitory control and frontal lobe function in ADHD (see section Introduction). It also replicates previous reports of altered ERP measures of prefrontal response control in children suffering from ADHD (Fallgatter et al, 2004) and a diminished NGA in adults with a suspected ADHD during childhood (Fallgatter et al, 2005). In the latter study, the diminished NGA was mainly caused by a more anterior centroid in the Go condition (Fallgatter et al, 2005); this is also in line with our results, as patients showed a significantly more anterior location of the Go centroid as compared with that in the control group (see also Baehne et al, 2009).

The significant interaction of the factors 'diagnosis' and 'genotype' furthermore indicates that the investigated VNTR had a differential effect on the NGA depending on the participants' diagnosis (ADHD patients $v s$ healthy controls). In the control sample, the SLC6A3 genotype had no significant effect, neither on the NGA itself nor on the position of the Go and NoGo centroid. In the ADHD group, however, we found a significant relationship between the genotypes and the NGA magnitude. This differential effect of the VNTR in patients and controls indicates that ADHD patients are particularly sensitive to genetic variants influencing dopaminergic transmission, possibly due to a generally altered dopaminergic state. In line with the finding of a relatively reduced NGA in the nine-repeat allele carriers of the ADHD group, a significant difference between patients and controls was only found in the 9/9 \& 9/10 genotype, with a significantly reduced mean NGA in the group of ADHD patients (see Figure 2). sLoreta source localization analyses indicated an origin of this effect in the anterior cingulate cortex, which showed significantly reduced NoGo-related activation in patients as compared with the controls within the subgroup of nine-repeat allele carriers. This finding fits well with previous data on reduced cingulate functioning in children and adults with a current or suspected previous ADHD diagnosis (Fallgatter et al, 2004, 2005).

While SLC6A3 is highly expressed in striatal areas, it is found in only low quantities in prefrontal and cingulate cortices (Sesack et al, 1998; Lewis et al, 2001). With respect to our finding of an effect of SLC6A3 on cortical activation within the group of ADHD patients (particularly within the inferior parietal cortex; cf. sLoreta results), SLC6A3 has nevertheless been assumed to have a crucial role in regulating the cortical signal-to-noise ratio in two ways. First, it appears to have a direct effect through its influence on prefrontal pyramidal neurons through regulation of DA volume transmission on the surrounding GABA-inhibitory neurons. Second, it influences the cortical signal-to-noise ratio indirectly through effects in the striatum, which regulates activity within the cortico-striato-thalamo-cortical pathway (Newman and Grace, 1999; also cf. Bertolino et al, 2006). Due to the involvement of striatal areas in motor regulation and response control, a particular effect of SLC6A3 on the Go-ERP (and related cortical sources) appears particularly plausible.

Our finding of a differential effect of the SLC6A3 genotype on the NGA has to be discussed in the light of genotypic consequences on a molecular, cellular, and neural network level. The effect of SLC6A3 has been extensively studied both in vitro and in vivo. However, in vitro studies (Mill et al, 2002; vanNess et al, 2005; Fuke et al, 2001; Miller and Madras, 2002; Greenwood and Kelsoe, 2003; Michelhaugh et al, 2001) have not yet been able to answer conclusively to what extent the SLC6A3 variants quantitatively affect SLC6A3 expression and DAT density, respectively. Single-photon emission computer tomography (SPECT) studies addressing this issue in vivo also led to partly inconclusive findings (Martinez et al, 2001; Heinz et al, 2000). Heinz et al (2000) reported a $22 \%$ reduction of DAT protein in the putamen of $9 / 10$ as compared with that in 10/10 genotype carriers, whereas Martinez et al (2001) found no differences in striatal radiotracer $\left(\left[{ }^{123} \mathrm{I}\right] \beta\right.$-CIT) binding. Two SPECT studies (van Dyck et al, 2005; van de Giessen et al, 2009) investigated this issue by measuring larger samples of healthy adult subjects. Both consistently showed that carriers of at least one nine-repeat allele exhibited increased striatal DAT availability. Interestingly, patients suffering from $\mathrm{ADHD}$ have also been assumed to show a striatal hypodopaminergic state due to heightened striatal DAT availability (Krause et al, 2000; see also Spencer et $a l, 2007)$. In line with these findings, Winsberg and 
Comings (1999) found that the 9/10 genotype was associated with a better response to methylphenidate (MPH) - which binds to DAT and inhibits DA transport - in children with ADHD.

Considering all these findings, we therefore favor the hypothesis of a relatively decreased DAT density in 10/10 allele carriers, despite inconsistent in vitro and in vivo findings. This would imply a relatively higher synaptic DA bioavailability in SLC6A3 10-repeat allele homozygotes as compared with that in nine-repeat allele carriers. The pattern of a reduced NGA in the 9/9 \& 9/10 allele carriers within our group of ADHD patients would also be in line with the assumption of an increased DAT density and decreased DA availability in the carriers of at least one nine-allele. Here, the SLC6A3 genotype-dependent relative increase of DA availability may underlie the 'endophenotypic rescue' within ADHD patients with the 10/10 genotype.

Our results are in accordance with longitudinal data provided by Barkley and co-workers who found thatcompared with 10/10 allele carriers - the 9/10 genotype was associated with more severe ADHD symptomatology and more unfavorable social and occupational outcomes. Interestingly, the authors furthermore report that some of their outcome measures were also impacted unfavorably by the $9 / 10$ genotype within their healthy control group (Barkley et al, 2006; see section Introduction). As this follow-up study comprised many different behavioral, psychological, and neuropsychological measures, one can conclude that the genotypic influence goes far beyond the categorical diagnosis of ADHD (as reported in association studies). Most interestingly, the seemingly adverse effects of the nine-repeat allele tended to become greater with increasing age of the subjects (with small effect sizes in children and moderate ones in adolescents). This finding strongly emphasizes the influence of genetics in the development of the individual and indicates that genetic effects may vary considerably throughout life time.

In summary, our findings are in accordance with studies favoring the nine-repeat allele as a risk allele for ADHD in adults (van Dyck et al, 2005; Franke et al, 2008, 2010), but not with studies favoring the 10-repeat allele that seems to be the risk allele for ADHD in children (Chen et al, 2003; Cook et al, 1995; Curran et al, 2001). Here the nine-repeat allele was associated with abnormalities in cognitive response control and related cortical activation in ADHD patients. Furthermore, neurophysiological differences between patients and controls were only apparent in one genotype group (9/9 \& 9/10), indicating the usefulness of combined approaches involving both the concept of endophenotypes (eg, behavioral inhibition) and imaging genetics (eg, influence of $S L C 6 A 3$ ). One factor possibly accounting for a differential association of SLC6A3 (and other genes) with ADHD or aspects of its symptomatology in children and adults might be changes in the requirements on the dopaminergic system throughout life. Spencer and co-workers, for example, found that the dopamine transporter density decreases during life with a numerically steeper slope for patients than control subjects (Spencer et al, 2005). From that point of view it is also plausible that in the later phases of life the genetic effect on dopaminergic activity might differ qualitatively and quantitatively from the effect in childhood. Thus, our endophenotypic association of the $9 / 9 \& 9 / 10$ genotype with a reduced NGA value in adults might not be contradictory to the association studies in children, but may depend on the subjects' age. However, although age might be the driving force, we cannot rule out influences of other factors that systematically differ between children and adult ADHD samples, that is, most notably the gender ratio and more extensive comorbidity rates in adult patients (Kessler et al, 2006). To further elucidate the development of $\mathrm{ADHD}$ throughout life longitudinal studies may provide helpful evidence concerning genetic influences, but are scarce up to now.

Limitations of our study include, although comparable with epidemiological studies (Kessler et al, 2006), a relatively high incidence of psychiatric (axis I-) comorbidities in the investigated ADHD sample (47\%), which might have led to a respective distortion of the results. Second, the gender ratio in our study differs from studies of children. Although the NGA seems to be independent of the gender in healthy subjects (Fallgatter et al, 1999b), it may explain some effects. Third, smoking behavior significantly differed between diagnostic groups (see section Materials and Methods), however, not between the SLC6A3 genotype groups $\left(\chi_{1}^{2}=0.76, p=0.38\right)$. Therefore, general group comparisons for neurophysiological findings might have been affected by differences in daily nicotine consumed, whereas the reported genetic findings should not be biased by this factor. Regarding the sample size, it would be desirable to investigate even larger samples to increase the reliability of the drawn conclusions. Therefore, multi-center studies should be conducted to increase the sample size.

\section{ACKNOWLEDGEMENTS}

This study was supported by the Deutsche Forschungsgemeinschaft (KFO 125, SFB TRR 58 C4) and the Bundesministerium für Bildung und Forschung (BMBF 01GV0605).

\section{DISCLOSURE}

The authors declare no conflict of interest.

\section{REFERENCES}

Almasy L, Blangero J (2001). Endophenotypes as quantitative risk factors for psychiatric disease: rationale and study design. Am J Med Genet 105: 42-44.

Baehne CG, Ehlis AC, Plichta MM, Conzelmann A, Pauli P, Jacob C et al (2009). Tph2 gene variants modulate response control processes in adult ADHD patients and healthy individuals. Mol Psychiatry 14: 1032-1039.

Barkley RA (1997). Behavioral inhibition, sustained attention, and executive functions: constructing a unifying theory of ADHD. Psychol Bull 121: 65-94.

Barkley RA (1998). Attention-Deficit Hyperactivity Disorder: a Handbook for Diagnosis and Treatment, 2nd edn. Guilford: New York.

Barkley RA, Smith KM, Fischer M, Navia B (2006). An examination of the behavioral and neuropsychological correlates of three ADHD candidate gene polymorphisms (DRD4 7+, DBH TaqI A2, and DAT1 $40 \mathrm{bp}$ VNTR) in hyperactive and normal children followed to adulthood. Am J Med Genet B Neuropsychiatr Genet 141: 487-498. 
Bellgrove MA, Hawi Z, Kirley A, Gill M, Robertson IH (2005). Dissecting the attention deficit hyperactivity disorder (ADHD) phenotype: sustained attention, response variability and spatial attentional asymmetries in relation to dopamine transporter (DAT1) genotype. Neuropsychologia 43: 1847-1857.

Bertolino A, Blasi G, Latorre V, Rubino V, Rampino A, Sinibaldi L et al (2006). Additive effects of genetic variation in dopamine regulating genes on working memory cortical activity in human brain. J Neurosci 26: 3918-3922.

Biederman J (2005). Attention-deficit/hyperactivity disorder: a selective overview. Biol Psychiatry 57: 1215-1220.

Biederman J, Faraone SV (2005). Attention-deficit hyperactivity disorder. Lancet 366: 237-248.

Bookheimer SY, Strojwas MH, Cohen MS, Saunders AM, PericakVance MA, Mazziotta JC et al (2000). Patterns of brain activation in people at risk for Alzheimer's disease. $N$ Engl J Med 343: 450-456.

Chen CK, Chen SL, Mill J, Huang YS, Lin SK, Curran S et al (2003). The dopamine transporter gene is associated with attention deficit hyperactivity disorder in a Taiwanese sample. Mol Psychiatry 8: 393-396.

Cheuk DK, Li SY, Wong V (2006). No association between VNTR polymorphisms of dopamine transporter gene and attention deficit hyperactivity disorder in Chinese children. Am J Med Genet B Neuropsychiatr Genet 141: 123-125.

Cook Jr EH, Stein MA, Krasowski MD, Cox NJ, Olkon DM, Kieffer JE et al (1995). Association of attention-deficit disorder and the dopamine transporter gene. Am J Hum Genet 56: 993-998.

Crosbie J, Perusse D, Barr CL, Schachar RJ (2008). Validating psychiatric endophenotypes: inhibitory control and attention deficit hyperactivity disorder. Neurosci Biobehav Rev 32: 40-55.

Curran S, Mill J, Tahir E, Kent L, Richards S, Gould A et al (2001). Association study of a dopamine transporter polymorphism and attention deficit hyperactivity disorder in UK and Turkish samples. Mol Psychiatry 6: 425-428.

Egan MF, Goldberg TE, Kolachana BS, Callicott JH, Mazzanti CM, Straub RE et al (2001). Effect of COMT Val108/158 Met genotype on frontal lobe function and risk for schizophrenia. Proc Natl Acad Sci USA 98: 6917-6922.

Ehlis AC, Reif A, Herrmann MJ, Lesch KP, Fallgatter AJ (2007). Impact of catechol-O-methyltransferase on prefrontal brain functioning in schizophrenia spectrum disorders. Neuropsychopharmacology 32: 162-170.

Epstein JN, Conners CK, Sitarenios G, Erhardt D (1998). Continuous performance test results of adults with attention deficit hyperactivity disorder. Clin Neuropsychol 12: 155-168.

Fallgatter AJ, Brandeis D, Strik WK (1997). A robust assessment of the NoGo-anteriorisation of P300 microstates in a cued Continuous Performance Test. Brain Topogr 9: 295-302.

Fallgatter AJ, Ehlis AC, Rosler M, Strik WK, Blocher D, Herrmann MJ (2005). Diminished prefrontal brain function in adults with psychopathology in childhood related to attention deficit hyperactivity disorder. Psychiatry Res 138: 157-169.

Fallgatter AJ, Ehlis AC, Seifert J, Strik WK, Scheuerpflug P, Zillessen KE et al (2004). Altered response control and anterior cingulate function in attention-deficit/hyperactivity disorder boys. Clin Neurophysiol 115: 973-981.

Fallgatter AJ, Jatzke S, Bartsch AJ, Hamelbeck B, Lesch KP (1999a). Serotonin transporter promoter polymorphism influences topography of inhibitory motor control. Int J Neuropsychopharmacol 2: $115-120$.

Fallgatter AJ, Mueller TJ, Strik WK (1999b). Age-related changes in the brain electrical correlates of response control. Clin Neurophysiol 110: 833-838.

Fallgatter AJ, Strik WK (1999). The NoGo-anteriorization as a neurophysiological standard-index for cognitive response control. Int J Psychophysiol 32: 233-238.
Faraone SV, Doyle AE (2000). Genetic influences on attention deficit hyperactivity disorder. Curr Psychiatry Rep 2: 143-146.

Faraone SV, Perlis RH, Doyle AE, Smoller JW, Goralnick JJ, Holmgren MA et al (2005). Molecular genetics of attentiondeficit/hyperactivity disorder. Biol Psychiatry 57: 1313-1323.

Franke B, Arias Vasquez A, Johansson S, Hoogman M, Romanos J, Boreatti-Hümmer A et al (2010). Meta-analysis of the SLC6A3/ DAT1 VNTR haplotype in adult ADHD suggests differential involvement of the gene in adult and childhood ADHD. Neuropsychopharmacoly 35: 656-664.

Franke B, Hoogman M, Arias Vasquez A, Heister JG, Savelkoul PJ, Naber $M$ et al (2008). Association of the dopamine transporter (SLC6A3/DAT1) gene 9-6 haplotype with adult ADHD. Am J Med Genet B Neuropsychiatr Genet 147: 1576-1579.

Fuke S, Suo S, Takahashi N, Koike H, Sasagawa N, Ishiura S (2001). The VNTR polymorphism of the human dopamine transporter (DAT1) gene affects gene expression. Pharmacogenomics $J$ 1: 152-156.

Gratton G, Coles MGH (1989). Generalization and evaluation of eye-movement correction procedures. J Psychophysiol 3: $1-50$.

Greenwood TA, Kelsoe JR (2003). Promoter and intronic variants affect the transcriptional regulation of the human dopamine transporter gene. Genomics 82: 511-520.

Heinz A, Goldman D, Jones DW, Palmour R, Hommer D, Gorey JG et al (2000). Genotype influences in vivo dopamine transporter availability in human striatum. Neuropsychopharmacology 22: 133-139.

Hünnerkopf R, Strobel A, Gutknecht L, Brocke B, Lesch KP (2007). Interaction between BDNF Val66Met and dopamine transporter gene variation influences anxiety-related traits. Neuropsychopharmacology 32: 2552-2560.

Jasper H (1958). Report of committee on methods of clinical exam in EEG. Electroencephalogr Clin Neurophysiol 10: 370-375.

Kessler RC, Adler L, Barkley R, Biederman J, Conners CK, Demler $\mathrm{O}$ et al (2006). The prevalence and correlates of adult ADHD in the United States: results from the National Comorbidity Survey Replication. Am J Psychiatry 163: 716-723.

Krause KH, Dresel SH, Krause J, Kung HF, Tatsch K (2000). Increased striatal dopamine transporter in adult patients with attention deficit hyperactivity disorder: effects of methylphenidate as measured by single photon emission computed tomography. Neurosci Lett 285: 107-110.

Langley K, Turic D, Peirce TR, Mills S, Van Den Bree MB, Owen MJ et al (2005). No support for association between the dopamine transporter (DAT1) gene and ADHD. Am J Med Genet B Neuropsychiatr Genet 139: 7-10.

Lehmann D (1987). Principles of spatial analysis. In: Gevins A, Remond A (eds). Handbook of Electroencephalography and Clinical Neurophysiology, Vol. 1: Methods of Analysis of Brain Electrical and Magnetic Signals. Elsevier: Amsterdam. pp 309-354.

Lehrl S (2005). Mehrfachwahl-Wortschatz-Intelligenztest MWT-B. Spitta Verlag: Balingen.

Leth-Steensen C, Elbaz ZK, Douglas VI (2000). Mean response times, variability, and skew in the responding of ADHD children: a response time distributional approach. Acta Psychol (Amst) 104: $167-190$

Levy F, Hay DA, McStephen M, Wood C, Waldman I (1997). Attention-deficit hyperactivity disorder: a category or a continuum? Genetic analysis of a large-scale twin study. J Am Acad Child Adolesc Psychiatry 36: 737-744.

Lewis DA, Melchitzky DS, Sesack SR, Whitehead RE, Auh S, Sampson A (2001). Dopamine transporter immunoreactivity in monkey cerebral cortex: regional, laminar, and ultrastructural localization. J Comp Neurol 432: 119-136.

Li D, Sham PC, Owen MJ, He L (2006). Meta-analysis shows significant association between dopamine system genes and 
attention deficit hyperactivity disorder (ADHD). Hum Mol Genet 15: 2276-2284.

Loo SK, Specter E, Smolen A, Hopfer C, Teale PD, Reite ML (2003). Functional effects of the DAT1 polymorphism on EEG measures in ADHD. J Am Acad Child Adolesc Psychiatry 42: 986-993.

Losier BJ, McGrath PJ, Klein RM (1996). Error patterns on the continuous performance test in non-medicated and medicated samples of children with and without ADHD: a meta-analytic review. J Child Psychol Psychiatry 37: 971-987.

Martin N, Scourfield J, McGuffin P (2002). Observer effects and heritability of childhood attention-deficit hyperactivity disorder symptoms. Br J Psychiatry 180: 260-265.

Martinez D, Gelernter J, Abi-Dargham A, van Dyck CH, Kegeles L, Innis RB et al (2001). The variable number of tandem repeats polymorphism of the dopamine transporter gene is not associated with significant change in dopamine transporter phenotype in humans. Neuropsychopharmacology 24: 553-560.

Michelhaugh SK, Fiskerstrand C, Lovejoy E, Bannon MJ, Quinn JP (2001). The dopamine transporter gene (SLC6A3) variable number of tandem repeats domain enhances transcription in dopamine neurons. J Neurochem 79: 1033-1038.

Mill J, Asherson P, Browes C, D'Souza U, Craig I (2002). Expression of the dopamine transporter gene is regulated by the $3^{\prime}$ UTR VNTR: evidence from brain and lymphocytes using quantitative RT-PCR. Am J Med Genet 114: 975-979.

Miller GM, Madras BK (2002). Polymorphisms in the $3^{\prime}$-untranslated region of human and monkey dopamine transporter genes affect reporter gene expression. Mol Psychiatry 7: 44-55.

Newman J, Grace AA (1999). Binding across time: the selective gating of frontal and hippocampal systems modulating working memory and attentional states. Conscious Cogn 8: 196-212.

Pascal-Marqui RD, Michel CM, Lehmann D (1994). Low resolution electromagnetic tomography: a new method for localizing electrical activity in the brain. Int J Psychophysiol 18: 49-65.

Pascual-Marqui RD (2002). Standardized low-resolution brain electromagnetic tomography (sLORETA): technical details. Method Find Exp Clin Pharmacol 24: 5-12.

Pascual-Marqui RD, Esslen M, Kochi K, Lehmann D (2002). Functional imaging with low resolution brain electromagnetic tomography (LORETA): a review. Method Find Exp Clin 24: 91-95.

Retz-Junginger P, Retz W, Blocher D, Weijers HG, Trott GE, Wender PH et al (2002). [Wender Utah rating scale. The shortversion for the assessment of the attention-deficit hyperactivity disorder in adults]. Nervenarzt 73: 830-838.

Rosvold HE, Mirsky A, Sarason I, Bransome ED, Beck LH (1956). A continuous performance test of brain damage. J Consult Psychol 20: 343-350.

Rubia K, Overmeyer S, Taylor E, Brammer M, Williams SC, Simmons A et al (1999). Hypofrontality in attention deficit hyperactivity disorder during higher-order motor control: a study with functional MRI. Am J Psychiatry 156: 891-896.

Sesack SR, Hawrylak VA, Matus C, Guido MA, Levey AI (1998). Dopamine axon varicosities in the prelimbic division of the rat prefrontal cortex exhibit sparse immunoreactivity for the dopamine transporter. J Neurosci 18: 2697-2708.

Simon V, Czobor P, Balint S, Meszaros A, Bitter I (2009). Prevalence and correlates of adult attention-deficit hyperactivity disorder: meta-analysis. Br J Psychiatry 194: 204-211.

Slaats-Willemse D (2003). Cognitive Endophenotypes of ADHD. Ponsen \& Looijen BV: Wageningen.

Smith AB, Taylor E, Brammer M, Toone B, Rubia K (2006). Task-specific hypoactivation in prefrontal and temporoparietal brain regions during motor inhibition and task switching in medication-naive children and adolescents with attention deficit hyperactivity disorder. Am J Psychiatry 163: 1044-1051.

Sonuga-Barke EJ (2002). Psychological heterogeneity in AD/HD-a dual pathway model of behaviour and cognition. Behav Brain Res 130: 29-36.

Spencer TJ, Biederman J, Madras BK, Dougherty DD, Bonab AA, Livni $\mathrm{E}$ et al (2007). Further evidence of dopamine transporter dysregulation in ADHD: a controlled PET imaging study using altropane. Biol Psychiatry 62: 1059-1061.

Spencer TJ, Biederman J, Madras BK, Faraone SV, Dougherty DD, Bonab AA et al (2005). In vivo neuroreceptor imaging in attention-deficit/hyperactivity disorder: a focus on the dopamine transporter. Biol Psychiatry 57: 1293-1300.

Todd RD, Huang H, Smalley SL, Nelson SF, Willcutt EG, Pennington BF et al (2005). Collaborative analysis of DRD4 and DAT genotypes in population-defined ADHD subtypes. J Child Psychol Psychiatry 46: 1067-1073.

van de Giessen EM, de Win MM, Tanck MW, van den Brink W, Baas F, Booij J (2009). Striatal dopamine transporter availability associated with polymorphisms in the dopamine transporter gene SLC6A3. J Nucl Med 50: 45-52.

van Dyck CH, Malison RT, Jacobsen LK, Seibyl JP, Staley JK, Laruelle $M$ et al (2005). Increased dopamine transporter availability associated with the 9-repeat allele of the SLC6A3 gene. J Nucl Med 46: 745-751.

Vandenbergh DJ, Persico AM, Uhl GR (1992). A human dopamine transporter cDNA predicts reduced glycosylation, displays a novel repetitive element and provides racially-dimorphic TaqI RFLPs. Brain Res Mol Brain Res 15: 161-166.

VanNess SH, Owens MJ, Kilts CD (2005). The variable number of tandem repeats element in DAT1 regulates in vitro dopamine transporter density. BMC Genetics 6: 55 .

Winsberg BG, Comings DE (1999). Association of the dopamine transporter gene (DAT1) with poor methylphenidate response. J Am Acad Child Adolesc Psychiatry 38: 1474-1477.

Wittchen H-U, Wunderlich U, Gruschwitz S, Zaudig M (1997). SKID-I. Strukturiertes Klinisches Interview für DSM-IV. Achse I: Psychische Störungen [SCID-I. Structured Clinical Interview for DSM-IV, Axis I-Disorders]. Göttingen: Hogrefe.

Yang B, Chan RC, Jing J, Li T, Sham P, Chen RY (2007). A metaanalysis of association studies between the 10-repeat allele of a VNTR polymorphism in the $3^{\prime}$-UTR of dopamine transporter gene and attention deficit hyperactivity disorder. Am J Med Genet B Neuropsychiatr Genet 144: 541-550. 